\title{
Entrainment of Oviposition under Various Light-Dark Cycles in the Domestic Fowl
}

\author{
Mitsuru Naito, Tetsuro Komiyama and Keijiro Nirasawa \\ National Institute of Animal Industry, \\ Tsukuba Norindanchi P. O. Box 5, Ibaraki 305
}

Timing of oviposition is controlled by an open period ${ }^{1,2)}$ during which an ovulatory release of LH (Luteinizing Hormone) may occur, and the phase of the open period is determined by an endogenous circadian clock $^{2}$. The circadian clock is mainly controlled by external light-dark signals ${ }^{2}$ in which onset of darkness is a main signal and onset of light is a subsidiary signal..$^{3,4)}$ LANSON STURKIE $^{5)}$ investigated the effect on oviposition of different dark periods and reported that 2.5 hour dark period was sufficient to alter the time of oviposition. CAIN and WILSON $^{6}$ ) reported that a 6 hour dark period had a great entraining capacity for oviposition. BHATTI and MoRRIs ${ }^{7)}$ reported that a 5 hour dark period was needed for entrainment of oviposition. These reports ${ }^{5 \sim 7)}$ were focused on the minimum dark period needed for entrainment of oviposition, but they did not agree on the time required. The present study was performed to determine the degree of entrainment of oviposition under various light-dark cycles.

\section{Materials and Methods}

The experiment was performed in a windowless house using 85 pullets of White Leghorn at 52 weeks of age. Birds were reared under 6L: $18 \mathrm{D}$ (6 hour light and 18 hour darkness, light-off at 16: 00 hours), $8 \mathrm{~L}: 16 \mathrm{D}, 11 \mathrm{~L}: 13 \mathrm{D}, 14 \mathrm{~L}: 10 \mathrm{D}, 18 \mathrm{~L}: 6 \mathrm{D}, 19$ L: 5 D, 19.5 L: 4.5 D, 20 L: 4 D, 21 L: 3 D, 22 L: 2 D, $23 \mathrm{~L}: 1 \mathrm{D}$ and $24 \mathrm{~L}: 0 \mathrm{D}$ successively for two weeks without changing the ligh-off time. Time of oviposition of each bird was recorded using the automated recording system ${ }^{8}$. The records of oviposition for the first one week of acclimation period ${ }^{4)}$ were not used. Mean oviposition time was calculated as the polar angle of the mean vector ${ }^{9)}$, in which each oviposition time was represented as a unit vector. Percentage of eggs laid in a modal 8-hour segment ${ }^{7)}$ which was the maximum proportion of eggs laid in segment of successive 8 hours was used as an index for measuring the entrainment of ovipositions.

\section{Results and Discussion}

The distribution of oviposition time is shown in Fig. 1. The mean oviposition time which is shown in Table 1 advanced gradually by changing from $6 \mathrm{~L}: 18 \mathrm{D}$ to $19.5 \mathrm{~L}$ : 

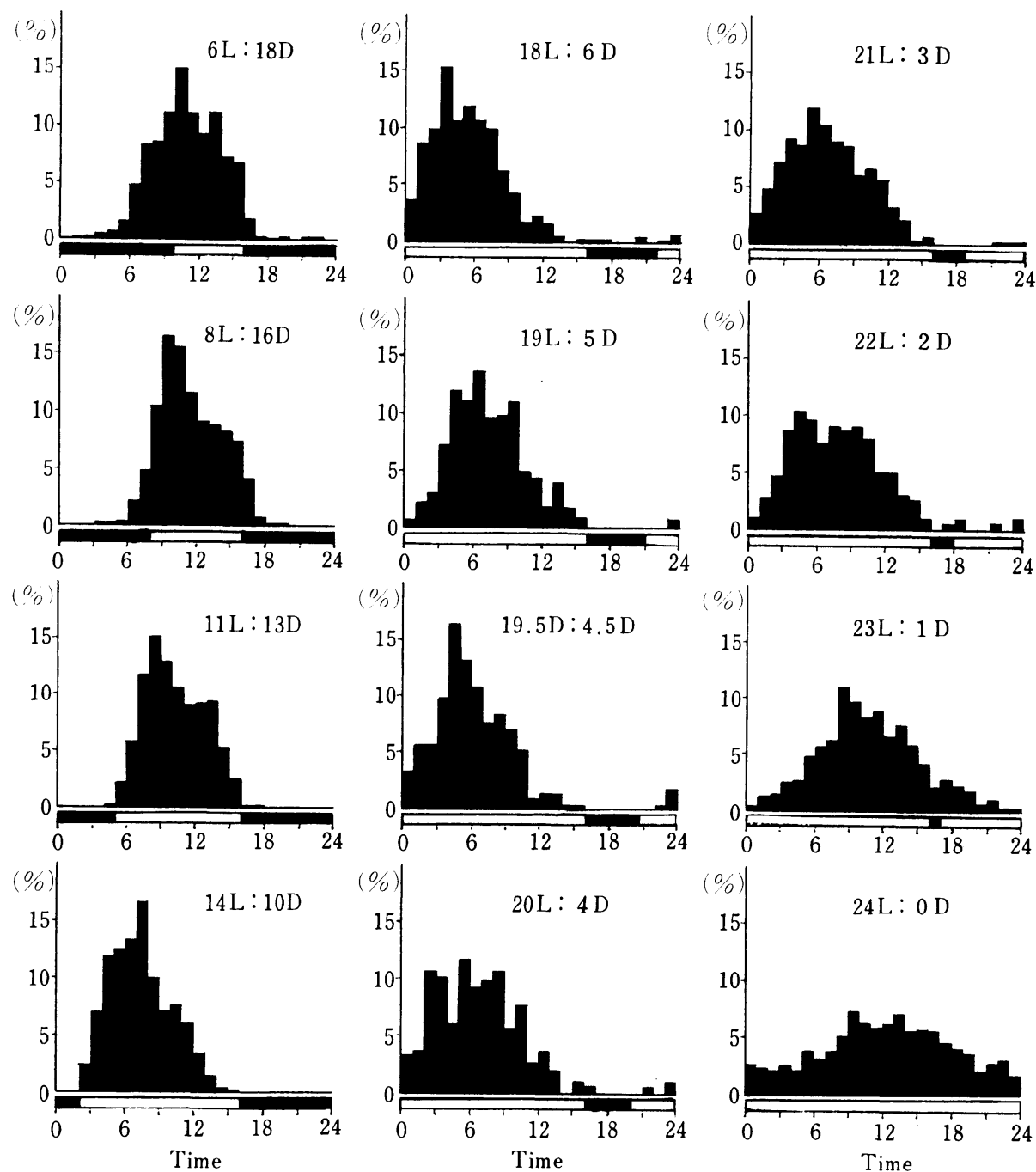

Fig. 1. Distribution of oviposition times of hens maintained under various light-dark cycles. Horizontal black bars show the dark period.

4.5 D, and retarded gradually from 19.5 L: 4.5 D irrespective of making the light-on time earlier. This retardation of mean oviposition time seems to be caused by the release from the control of the light-dark cycle for entrainment of oviposition as shown in the value of the percentage of eggs laid in the modal 8 hours (Table 1). The value presenting the entrainment of oviposition increased gradually by changing from $6 \mathrm{~L}$ : $18 \mathrm{D}$ to $11 \mathrm{~L}: 13 \mathrm{D}$, and decreased gradually from $11 \mathrm{~L}: 13 \mathrm{D}$ to $24 \mathrm{~L}: 0 \mathrm{D}$. The value under 19L: $5 \mathrm{D}$ was about $80 \%$ which was fully entrained, and it was in agreement with the results of CAIN and WILsON ${ }^{6)}$ and Bhatti and Morris ${ }^{7)}$.

Based on these results, the following equation is calculated.

$$
\mathrm{Y}=55.676+6.045 \mathrm{X}-0.256 \mathrm{X}^{2} \quad(\mathrm{R}=0.97, \mathrm{P}<0.01)
$$

where $\quad \mathrm{Y}$ : percentage of eggs laid in the modal 8 hours 
Table 1. Time of oviposition under various light regimens

\begin{tabular}{ccc}
\hline Light regimen* & $\begin{array}{c}\text { Mean oviposition time } \\
\text { (Clock hour) }\end{array}$ & $\begin{array}{c}\text { Percentage of eggs laid } \\
\text { in the modal } 8 \text { hours }\end{array}$ \\
\hline 6L: 18D & $11: 02$ & 82.3 \\
8L $:$ 16D & $11: 18$ & 87.4 \\
$11 \mathrm{~L}:$ 13D & $10: 01$ & 90.4 \\
$14 \mathrm{~L}:$ 10D & $07: 17$ & 87.5 \\
$18 \mathrm{~L}:$ 6D & $05: 06$ & 83.7 \\
19L $:$ 5D & $07: 08$ & 79.7 \\
$19.5 \mathrm{~L}:$ 4.5D & $05: 43$ & 78.9 \\
20L $:$ 4D & $06: 19$ & 73.8 \\
21 L : 3D & $06: 31$ & 71.9 \\
22L $:$ 2D & $07: 21$ & 71.5 \\
23L $:$ 1D & $10: 25$ & 64.7 \\
24L $:$ 0D & $12: 37$ & 49.4 \\
\hline
\end{tabular}

* Light off; $16: 00$ hours

\section{$\mathrm{X}$ : hours of darkness in a light-dark cycle \\ $\mathrm{R}$ : multiple correlation coefficient}

This equation fits to the data quite well, so that the degree of entrainment of oviposition under light-dark cycles should be considered continuous relation between the two rather than the threshold. Using this equation percentage of eggs laid in the modal 8 hours shows the maximum value in around $12 \mathrm{~L}: 12 \mathrm{D}$. The circadian clock which controls the phase of the open period is stimulated by each of onset of darkness and light. Here it is assumed two circadian oscillators ${ }^{10)}$, one is entrained by onset of darkness and the other by onese of light, and these oscillators control the phase of the open period. The degree of entrainment depends on the phase angle between the two oscillators and the greatest entrainment is obtained in $12 \mathrm{~L}: 12 \mathrm{D}$ because in this case the two oscillators synchronize with each other. The results of this experiment are consistent with above assumption. However, BHATTI and MORRIS ${ }^{7)}$ reported that entrainmeat on the 19L: $5 \mathrm{D}$ was similar to that on the $14 \mathrm{~L}: 10 \mathrm{D}$. In this experiment these two treatments were not the same for entrainment of oviposition. The discrepancy between the results is unclear, so that further studies are needed to ascertain the role of onset of darkness and onset of light on entrainment of oviposition as well as the above assumption.

\section{Literature}

1) Fraps, R. M. (1954) Neural basis of diurnal periodicity in release of ovulation-inducing hormone in fowl: Proc. Nat. Acad. Sci., U. S. A., 40: 348-356.

2) Morris, T.R. (1973) The effects of ahemeral light and dark cycles on egg production in the fowl: Poult. Sci., 52: 423-445.

3) Bhatti, B. M. and T. R. MorRis (1978) The relative importance of sunrise and sunset for entrainment of oviposition in the fowl: Brit. Poult. Sci., 19: 365-371.

4) Naito, M., T. Ueno, T. Komiyama and Y. Miyazono (1980) Oviposition times under various light-dark cycles in the domestic fowl: Japan. Poult. Sci., 17: 146-150. 
5) Lanson, R.K. and P.D. Sturkie (1958) Effect of the length of the dark and light periods on time of oviposition: Poult. Sci., 37: 1219.

6) CAIN, R. J. and W. O. Wilson (1974) The influence of specific environmental parameters on the circadian rhythms of chickens: Poult. Sci., 53., 1438-1447.

7) Bhatti, B. M. and T.R. Morris (1978) Entrainment of oviposition in the fowl using light-dark cycles: Brit. Poult. Sci., 19: 333-340.

8) Ueno, T., Y. Miyazono, T. Komiyama and S. Ohtsuka (1978) Time of oviposition and ovipository cycle in hens: Bull. Nat. Inst. Anim. Ind., 33: 87-91.

9) Batschelet, E. (1965) Statistical methods for the analysis of problems in animal orientation and certain biological rhythms: Amer. Inst. Biol. Sci., Wash., U. S. A.

10) FARNeR, D.S. (1980) Endogenous periodic functions in the control of reproductive cycles: In Biological Rhythms in Birds (Y. TAnaBe et al. eds.): 123-138, Japan Sci. Soc. Press, Tokyo/ Springer-Verlag, Berlin. 